\title{
ZUR SEMIOTIK EINIGER MUSIKINSTRUMENTE (VORLÄUFIGE PROBLEMSTELLUNG)
}

\author{
Vilmos Voigt \\ Lehrstuhl für Folkloristik, Eötvös Loránd Universität, Budapest \\ H-1088 Budapest, Múzeum körút 6-8. \\ voigtbudapest@gmail.com
}

\begin{abstract}
Towards a Semiotics of Musical Instruments. Outlines of the Problem. In spite of the promising recent development of comparative musicology (also including the study of musical instruments) and of semiotics (also including musical semiotics), there is no summarizing attempt to describe and analyze the "signs on musical instruments" phenomena, i.e. carved or painted parts of the instruments. The zoomorphic and anthropomorphic construction and forms of musical instruments, and of their parts, is a wide-ranging field of study. The paper shows some examples of ancient and folk music instruments, by using the common (Peircian) terminology in describing their signs in the proper sense of the word. Animal shells used as bodies of instruments, snake- and dragon-formed instruments, amorous heads on string instruments, human heads and devilish forms of bagpipes, paintings on piano's wooden cases, emblems or coats of arms of the builders of the instruments - just there are some cases of signs of musical instruments. There are further allusions to musical signs as well.
\end{abstract}

Keywords: semiotics, musical instruments, anthropomorphic and zoomorphic details of musical instruments, signs, symbols, iconography of music

\section{Zu einem Themengespräch mit Gy. Martin zurückführend}

In den letzten Jahrzehnten haben die internationale und die ungarische Musikwissenschaft groß angelegte Synthesen ins Leben gerufen. In diesen ist auch viel von der gesellschaftlichen Benutzung der Musik die Rede. Auch über alte und heutige Musikinstrumente sind gute Handbücher erschienen. ${ }^{1}$ Ähnlich wichtige Überblicke brachte die internationale Semiotik hervor und in diesen Handbüchern und Lexika kommt auch die Semiotik der Musik zur Sprache. ${ }^{2}$ Bereits in den 1960er Jahren erwies sich auch die vielfältige Untersuchung

${ }^{1}$ Siehe z. B. Midgley 1978; Baines 1992; Sadie 1984 usw. Siehe auch: Schaffner 1936 und MarcelDubois 1980. VAN DeR MeER 1983 erschien auch in ungarischer Übersetzung (1988).

${ }^{2}$ Siehe z. B. Posner-Robering-SEbeok 1997-2004; SEBeok-Danesi 2010. Theoretisch besonders wertvoll ist: Nöth 2000 bzw. Kaden-Brachmann-Giese 1998. Zur einfachen Einleitung: Voigt 2008. 
der vielerlei Musik als eines der verheißungsvollsten Forschungsgebiete der Semiotik. In den letzten Jahrzehnten erschienen - in erster Linie dank der Organisationsfähigkeit des Professors Tarasti aus Finnland ${ }^{3}$ - regelmäßig musiksemiotische Monographien, Kongressberichte und Überblicke. Diese beschäftigen sich häufig mit der Musik alter und außereuropäischer Kulturen, mit dem ,Inhalt“ und mit der Semantik der Musikwerke. Als vornehmste Beispiele können wir gleichwohl die Opern und die Programm-Musik anführen. Hierbei wie in der Musikgeschichte wurde den auch unter zeichentheoretischem Aspekt wichtigen Themen der Beschreibung und Systematisierung der Töne (Notenbeschreibung, System und Entwicklung der Tonarten usw.) viel Aufmerksamkeit gewidmet. Weniger Aufmerksamkeit wurde meines Wissens den Musikinstrumenten und besonders den an diesen befindlichen Zeichen (wie z. B. zoomorphen bzw. anthropomorphen Bestandteilen der Instrumente, Malerei und Intarsien auf dem Instrumentendeckel usw.) zuteil.

Wir wissen es sehr wohl auch aus Erfahrung, dass die Musikinstrumente nicht nur den akustischen Gesetzmäßigkeiten des eng gefassten Praktizismus folgen. Sehr häufig sind es sorgfältig ausgearbeitete Meisterwerke des Kunstgewerbes, mit vielen ästhetischen Griffen, deren Gestalt und Form gleichermaßen auch unter zeichentheoretischem Aspekt charakterisiert werden können. Die Zahl der wirklich semiotisch fertiggestellten derartigen Studien ist jedoch unglaublich gering, als hätte dieses Thema kaum jemanden unter den Musikforschern bzw. Zeichenforschern interessiert. Auch die Kunstgeschichte kann vieles zu diesem Thema beitragen. ${ }^{4}$

Die historischen-bildlichen Darstellungen der glücklicherweise bis zum heutigen Tag in großer Zahl erhalten gebliebenen jahrhundertealten Musikinstrumente haben die Aufmerksamkeit der Forscher aus mehreren Disziplinen erregt. ${ }^{5}$ Dies ist sowohl für Kunsthistoriker als auch für Musikhistoriker ein Quellenmaterial von entscheidendem Wert. ${ }^{6}$ Wir können im Hinblick auf die Entwicklung der Instrumente, mitunter auf die Art, wie auf ihnen gespielt wurde, und sogar auf das gesellschaftliche Umfeld manchmal geradezu mit der Präzision eines Farbfilms die Auskunft erhalten: Wer, wann und für wen auf diesen gezierten Instrumenten spielte. Auf Grund der Kenntnis der von der historischen Ikonographie offengelegten Konventionen können wir sogar auf die „künstlerische Semantik“ dieser Darstellungen schlussfolgern - wobei wir zu bedenken haben, dass nicht die jeweiligen Bilddetails wichtig oder genau treffend sind, sondern die Einstellung des ganzen Kunst- oder Kunstgewerbewerks unter musiksemiotischem Aspekt eine wichtige Tatsache ist. Wenn wir an solche allgemein bekannten musik- oder kunsthistorischen Bravur-Aufsätze denken wie die Interpretationen der Darstellungen der Teufelsmusik, „musizierender Engel“7 bzw. „östlicher“" oder „primitiver“", eventuell „,bäuerlicher“ Musikinstrumente, dann wird ersichtlich, mit welchem frappierenden kulturhistorischen Beweismaterial wir hier zu tun haben.

${ }^{3}$ Siehe besonders: TARASTI 1994.

${ }^{4}$ Siehe zusammenfassend: WinTERNITZ 1967.

${ }^{5}$ Siehe die reichen Musikinstrumentensammlungen und -museen z. B. in Berlin, Leipzig, Kopenhagen, Brüssel, Paris, Wien, Rom, Milano, London, Moskau, Prag usw. In Budapest wurde die neue Ausstellung „Musikinstrumente in Ungarn“ im Musikhistorischen Museum im Oktober 2012 geöffnet.

${ }^{6}$ Siehe z. B. das Prachtwerk von Wade-MatThews 2009 (früher: 2000).

${ }^{7}$ Sie z. B. das berühmte Tafelbild von Hans Memling: Musizierende Engel (Antwerpen, um 1480). 
Dennoch beschäftigen wir uns an dieser Stelle nicht mit diesem allgemein bekannten Fragenkomplex. Vielmehr werden wir Musikinstrumente vorstellen, deren Gestaltung oder die Formung einzelner Teile als „,symbolisch“ - genauer gesagt „,zoomorphisch“ oder „anthropomorphisch“ - betrachtet werden können. Da wir diese „symbolische“ Gruppe der Musikinstrumente sogleich auch präziser umreißen werden, sei hier lediglich der Hinweis gestattet, dass besonders die Nachahmung von Tier- und Menschenköpfen, der Gestalt von Fabelwesen nachempfundene Musikinstrumente in verschiedenen Epochen von vielerlei Kulturen zu beobachten sind. Auch kulturgeschichtlich ist es ein weites Feld: Die beim einfachen Volk gebräuchlichen einfachsten Knochen- und Toninstrumente ${ }^{8}$ können ebenso tiergestaltig sein (z. B. die vogelförmigen Tongefäßpfeifen), wie die wunderschönen plastischen Menschenköpfe an den von den besten Instrumentenbauern für den Hochadel ins Werk gesetzten Streichinstrumenten. ${ }^{9}$ In jeder Hinsicht sind beide als hochwertige ,semiotische“ Kunstwerke zu betrachten.

Da es sich um ein weltweit vorhandenes Phänomen handelt - d. i. zeichenhafte Kunstformen auf den Musikinstrumenten -, behandele ich aus diesem Komplex nur einige herausgehobene Beispiele. Ich könnte lang aufzählen, was ich hier warum nicht detailliert vorstellen werde. Was die semiotische Terminologie betrifft, folge ich einfach der bekannten Zeichentypologie von Charles Sanders Peirce.

Es ist allgemein bekannt, dass bereits bei den primitiven und alten Völkern die in der Natur vorgefundenen „,fertigen“ Formen als Instrumente verwendet werden. Hörner werden aus Schnecken und Muscheln hergestellt; Panzer von Schildkröten und ähnlichen Panzertieren halten auch als Tonkasten von Saiteninstrumenten her (Abb. 1). In solchen Fällen ist das Musikinstrument natürlich ein (An)Zeichen des ursprünglichen Tieres. Genauer - obgleich es der semiotischen Terminologie zufolge als Index-Zeichen betrachtet werden kann - wirkt es häufig schon als Ikon-Zeichen. Und es braucht nicht einmal wortwörtlich formuliert zu werden, dass beim Erklingen dieser Instrumente „das Tier selbst“ die Töne von sich gibt - um den Ursprung der Form wissen Interpreten und Zuhörer gleichermaßen. Das lässt sich leicht belegen. Tschurunga (Wirrholz) wird auf vielen Erdteilen als bull-roarer (Stier-Gebrüll) bezeichnet.

Die Denotation und Konnotation der „Tiere als Musikinstrumente“ führen aber weiter. Altchinesische Glocken und Gongs zeigen nicht nur die Tierformen vor (Abb. 2), auch Tiere (wie z. B. Tiger) werden am Rande des Instruments als konnotative Zeichen vorgezeigt (Abb. 3).

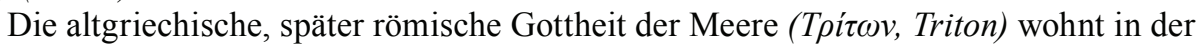
Tiefe des Meeres, bricht jedoch, mal zu eigenem Vergnügen, mal auf Befehl des obersten Meeresgottes Poseidon, häufig zu Rundreisen durch die Meere auf. Seine Begleiter sind Seerosse und etliche Wasserungeheuer. Seine laute Trompete ist eine schneckenförmige Seemuschel, mit der er mal dem tobenden Meer befiehlt, sich zu glätten, mal die Feinde der olympischen Götter (z. B. die Giganten) mit lautem Getöse abschreckt. Eben darum

${ }^{8}$ Z. B. die okarinaähnliche, irdene Gefäßflöte ist ein uralter Instrumententyp; man kann sie von Assyrien über Zentral- und Ostasien bis nach der Südsee und Amerika bzw. auf der anderen Seite bis nach Westafrika verfolgen. Siehe: SACHS 1930: 76.

${ }^{9}$ Siehe besonders die „lira da braccio“-Instrumente des 16. Jahrhunderts in Venedig. Die Monographie davon: JONES 1995. 
lautet sein Beiwort concha canens, canorhs, ' $\eta \pi \dot{\tau} \tau \eta$ s. Auch in Altamerika, z. B. in dem vorkolumbischen Mexiko, hat man Muscheltrompeten gespielt. Im alten China riefen $\mathrm{Mu}-$ scheltöne für Andacht an.

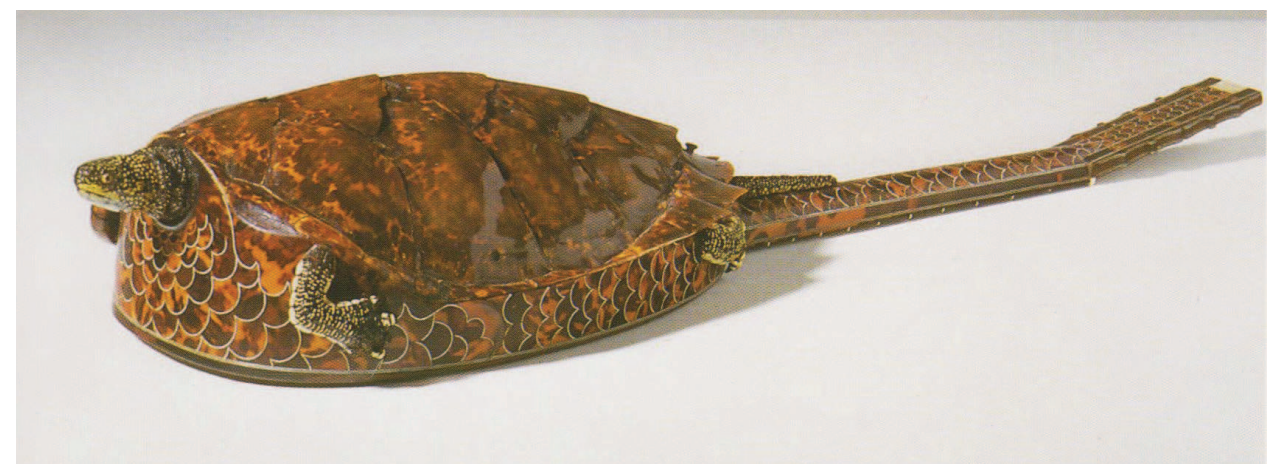

Abb. 1. Gitarre in Schildkrötenpanzerform, verfertigt von Jean Voboam. Paris, 1693

Eine auffallende Gestalt von Blasinstrumenten ist nicht selten die der Schlange (Abb. 4) oder des Drachens (Abb. 5). Wie es auch durch die Formung des Serpents (und sogar der ihm ähnlichen Bassklarinette) angedeutet wird, ist eine derartige gestaltgemäße Semiose auch in der heutigen Musikkultur nicht unbekannt.

Wohl wissen wir auch, dass sehr alte Musikinstrumente mit Geräten, die auch anderen praktischen Zielen (z. B. Jagd, Milchwirtschaft) dienen, in Verbindung stehen - wie zum Beispiel der Zupfbogen/Streichbogen oder auch der Dudelkrug und seine Verwandten. In solchen Fällen ist das Musikinstrument in semiotischem Sinne ein Signal des betreffenden Gegenstandes oder Gerätes. Heutzutage verdeutlichen die als Musikinstrumente verwendeten Löffel und Kämme, dass diese Praxis fortbesteht. An dieser Stelle müssen wir jedoch von der eingehenden Vorstellung dieses Zeichentyps absehen, obgleich ein „Musikinstrument“ als Gerät oder Gegenstand auch eine andere praktische Verwendung hat (wie z. B. eine Hirtenpeitsche), sowohl musikalisch als auch semiotisch außerordentlich interessant ist auch für eine Theorie der Zeichen.

Was ist in diesem Fall sein Signal und wessen Signal ist es? Hierbei denken wir nicht lediglich in Leopold Mozarts Kleinsymphonie Die Bauernhochzeit (1755) an die abgeführte Bühnenpistole, die knarrenden Ratschen, sondern zum Beispiel an die Kirchenglocken (die durch die feine Musik in der Regel nur nachgeahmt werden, ohne selbst zu erklingen). Selbst die experimentelle Musik des 20. Jahrhunderts, wie z. B. Olivier Messiaens „Vogelmusik“ (siehe ihre Anfänge: Réveil des oiseaux /1953/ und Oiseaux exotiques /1956/, schließlich ihre Vollendung: Catalogue d'oiseaux /1956-1958/), kann verdeutlichen, wie kompliziert das musikalische ,Zeichen“ wird, wenn es nicht aus der autonomen Hochmusik stammt und in ihm Nicht-Musik als Musik, Nicht-Musik-Instrument (hier z. B. die Vögel) als Musikinstrument fungieren. 


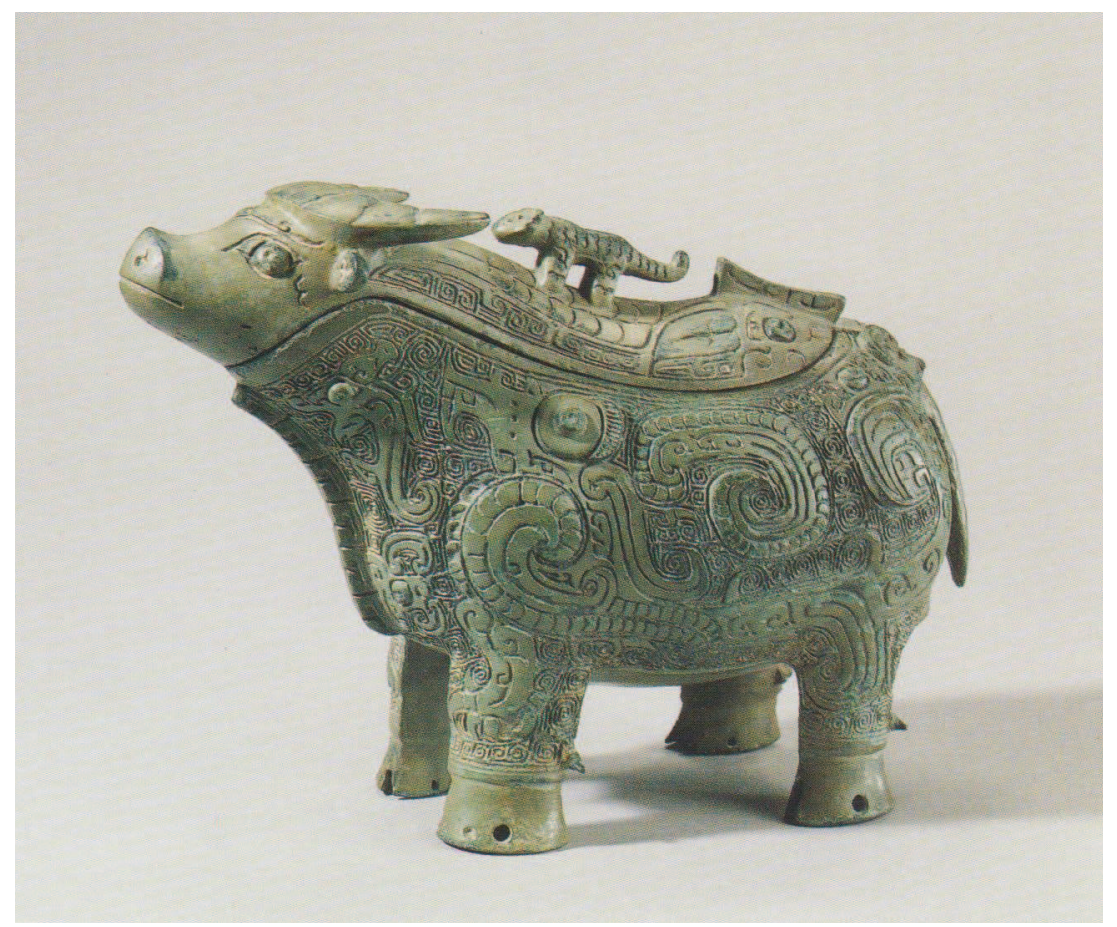

Abb. 2. Bronze Gong-Vase in Büffelform, mit eidechsenartigem Handgriff. China, 12.-11. Jh. v. Chr.

Dieser breite Fragenkomplex lässt sich im Wortgebrauch der Semiotik immer mit der Kategorie des Signals kennzeichnen. So werden auch jene Zeichen als solche genannt, die von den Zeichenträgern nicht zu trennen sind. Da das „Signal“ ein auch in der engen Musikwissenschaft allgemein bekannter Funktionsbegriff ist - und der in der Semiotik gewohnten Form und Inhalt gar nicht so fern ist -, wäre es sinnvoll, sich mit dieser Erscheinung auch gesondert zu beschäftigen.

Wenn zum Beispiel eine „Hirtenflöte“ oder ein „Bauerndudelsack“ erklingt, signalisiert das Instrument nicht nur einen musikalischen Stil, sondern auch die ursprüngliche Gesellschaftsschicht selbst, die bestimmte Kultur, welcher es entstammt. Natürlich wird die Signalisierung reicher, wenn das Instrument zugleich eine sowohl musikalische als auch der eigentlichen Subkultur entsprechende Melodie zu Gehör bringt, die dem adäquaten gesellschaftlich-kulturellen Umfeld zugeordnet werden kann.

Der Klang der Militärkapelle, des Nebelhorns, aber auch die „Musik“ der Dampflokomotive ist in dieser Rolle gleichermaßen bekannt. Hierbei kann freilich mit mehrfacher Übertragung und Bedeutungswechsel gerechnet werden. Die Wenigen wissen, dass die Fortsetzung von Arthur Honeggers berühmter Eisenbahnmusik (Pacific 231 - aus 1923) das Rugby (1928) ist, wobei in dem Letzteren jedoch keine solche direkte Klangquelle aufzufinden ist, die das vorgeführte Ereignis eindeutig signalisieren würde. All dies verdiente freilich eine selbstständige Abhandlung auch im Rahmen der Musiksemiotik. 


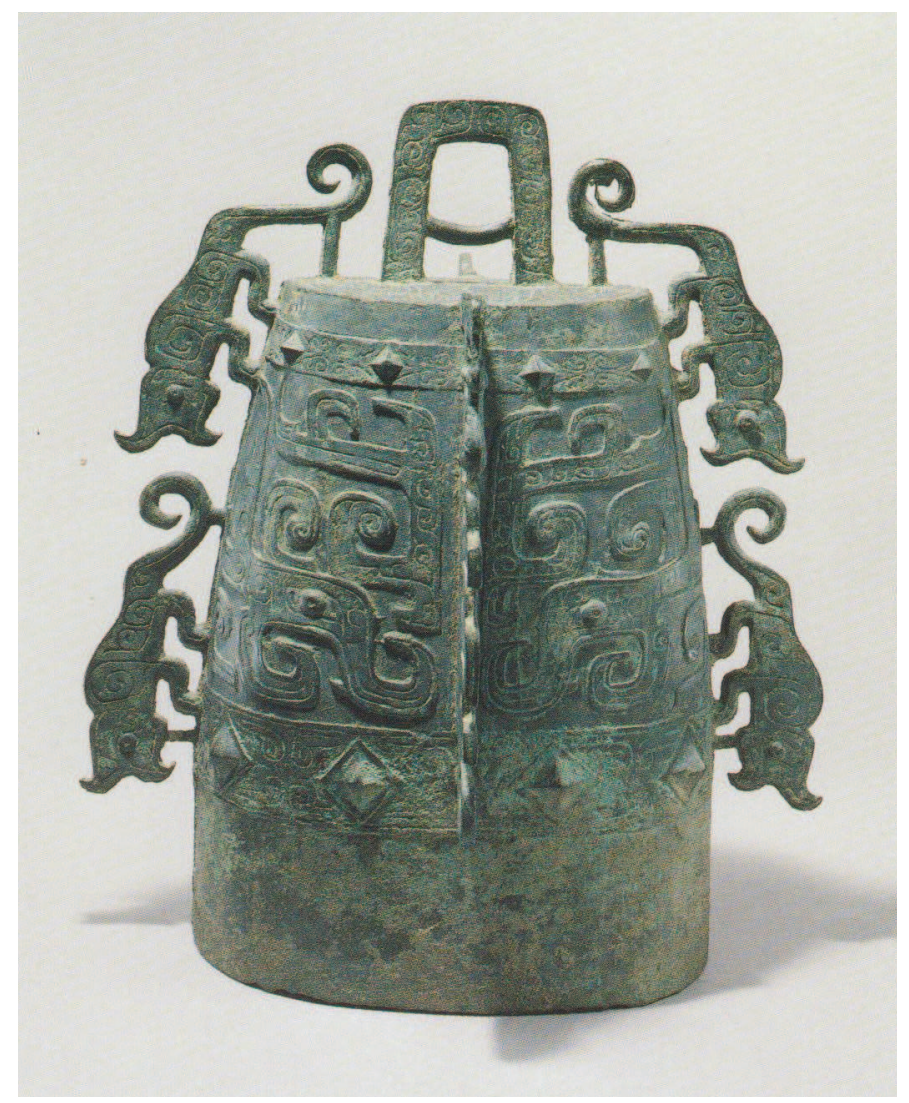

Abb. 3. Chinesische Bronzeglocke mit vier Tigerfiguren, 10. Jh. n. Chr.

Im Hinblick auf die eigentliche Semiotik der Kultur warten jene Fakten mit den größten Lehren auf, bei denen eine Art des Signalgebrauchs über eine relativ große Zeit und in vielen Einzelfällen auftritt. Da lassen sich der geschichtsgebundene Gebrauch der Signale und Signalgruppen bzw. ihre Wandlungen unmittelbar untersuchen.

Ein gutes Beispiel dafür, auf welches wir jedoch ebenfalls nicht eingehen werden, ist die Geschichte der in mit Deckeln versehenen Holzschränken untergebrachten Musikinstrumente. Dazu gehört nicht nur das Klavier selbst, sondern auch dessen mehr oder weniger entfernte Verwandte (Virginal, Cembalo, Clavecin, Spinett usw.). Auch an den Vorder- und Hinterseiten dieser großformatigen Holzkasten prangen häufig Verzierungen (und gar nicht immer in der ornamentalen Art der Tischler), die den schönsten Möbelstücken zur Ehre gereichen würden. An der Innenseite des aufklappbaren Deckels (seltener auch außen) sind oft üppige Gemälde mit vielen Gestalten bzw. mit Landschaftsbildern zu sehen (Abb. 6). Ihre Thematik ist wahrhaftig abwechslungsreich: Sie zeigen Genrebilder, mythologische und religiöse Szenen oder eben Wappen, Zeichen, Datierungen, Initialen usw., die auf den Auftraggeber oder den Instrumentenbauer hinweisen. Die prachtvolle 
Dekorativität derartiger Instrumente, die die „Schönheit“ der Musik bekräftigt, ließ im 19. Jahrhundert erheblich nach, tritt jedoch heutzutage - eher an die Popmusik geknüpft erneut in Erscheinung. Eine eingehendere semiotische Untersuchung der Thematik solcher „Gemälde“ auf den Musikinstrumenten wäre überaus lehrreich, weil sie die Intersemiotizität zweier Künste betonen. Doch ist dieses künstlerisch-ikonographische Material meines Wissens noch nicht einmal inventarisiert worden.

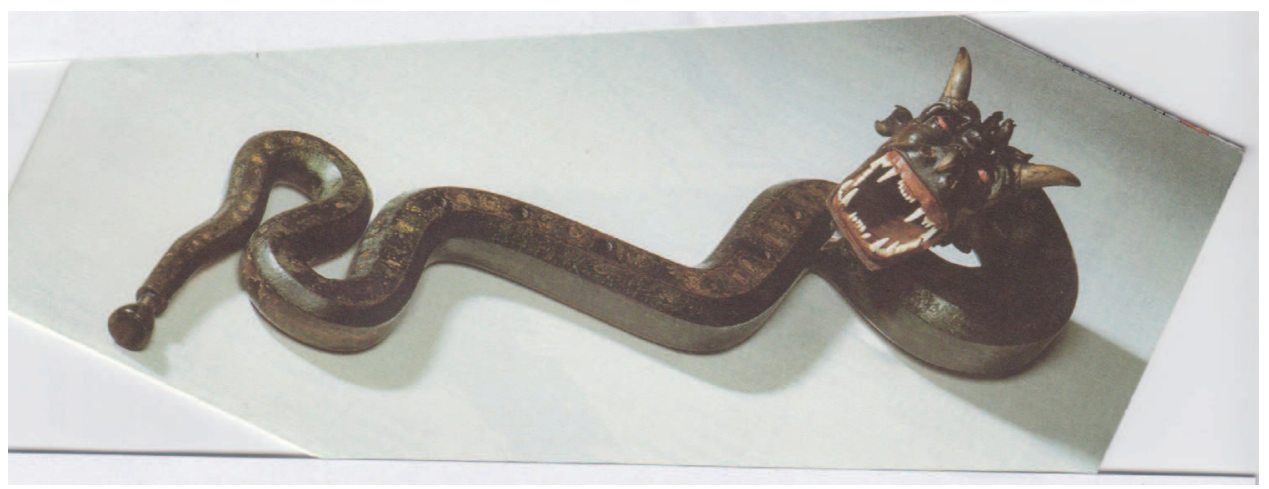

Abb. 4. Basskornett in Schlangenform mit fabelhaftem Teufelskopf. Italien, 16. Jh.

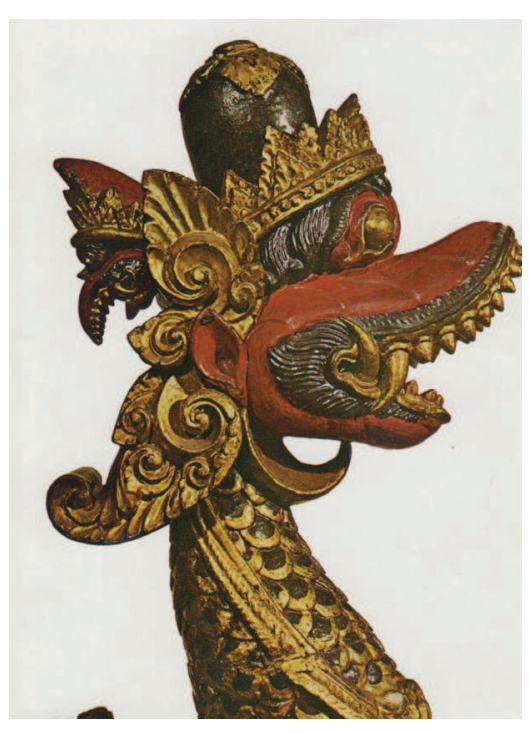

$A b b$. 5. Kopfteil eines Gamelaninstruments (gong ageng) aus Java (Indonesien) mit Drachenkopfdarstellung
Will man solche Signalzeichen in einem Inventarium (Datenbank) zusammenstellen, wäre es die einfachste Vorgehensweise, die je nach Material, Form und Herstellungsart gut zu differenzierenden Musikinstrumente sich einzeln vorzunehmen. Der oberflächliche Betrachter könnte annehmen, dass alle Musikinstrumente auf diese Weise gleichermaßen zu Zeichenträgern werden könnten. Dem ist aber nicht einfach so: Die Zeichen mancher bestimmten Instrumente wiederholen sich paradigmatisch, also unzählige Male, während sie bei anderen Instrumenten ganz anders oder ganz selten erscheinen.

An Trommeln oder gewissen Militärmusikinstrumenten referieren diese Zeichen die Heraldik der Herrscher oder der Staaten: Es kommen Wappen, Flaggen, Schutzheilige oder Devisen vor. ${ }^{10}$ Diese Zeichen entsprechen etwa denen, die an den Staatsgrenzschranken und Wachhäuschen, auf Goldmünzen oder am Schiffsschnabel (heute auch

${ }^{10}$ Man sieht auf Zugtrompeten kleine Fahnen mit heraldischen Lilien auf dem Gemälde „Die AdimariHochzeit“, Florenz, um 1450. Siehe: Buchner 1995, Farbbild Nr. 87 auf Seite 82. 


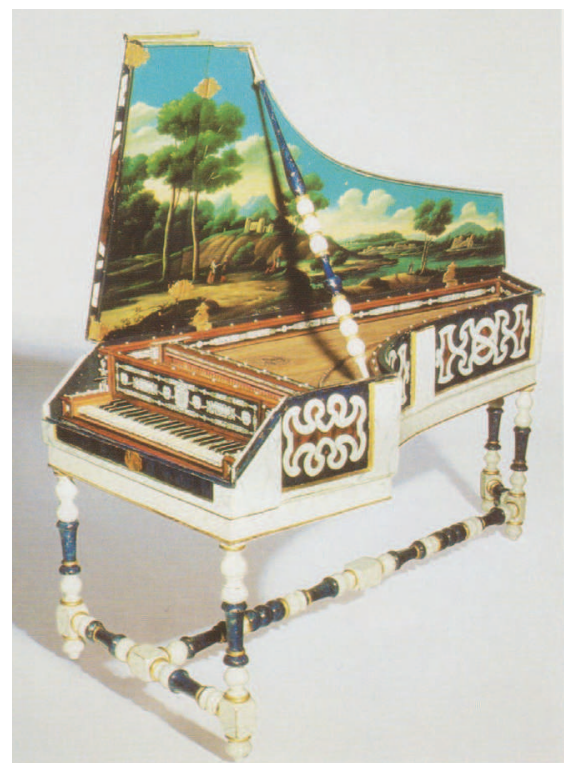

Abb. 6. Clavecin mit Landschaftsbild, gebaut von Faby (Hercule Pepoli?). Bologna, 1677 an Flugzeugen) zu beobachten sind. Obwohl sie in eigentlichem Sinne des Begriffs Zeichen sind (in der Regel, kulturgeschichtlich gesehen $\mathrm{Em}$ bleme und Symbole), gehören sie dennoch nicht automatisch zur Musiksemiotik, sondern zur Semiotik der Hoheitszeichen und überhaupt der Staatsinsignien $(A b b .7)$. Ihre Verwandten sind heutzutage eher an den Registrationsschildern der Automobile, denn in der Welt der Musikzeichen zu finden.

Auf vielen heutigen Musikinstrumenten ist das Markenzeichen (Brandmarke) der Produktionswerkstatt oder der Fabrik zu sehen - häufig auch in einer reich verzierten Version. Dies kennzeichnet vor allem die kostspieligeren, festlichen, neuartigen, im show-business verwendeten Tonträger. Das sind jedoch ebenfalls keine Musikzeichen, viel mehr Markenzeichen, die sich von den Markenzeichen z. B. an Schreibmaschinen oder Fahrrädern nicht allzu sehr unterscheiden. Die Markenzeichen der Instrumen-

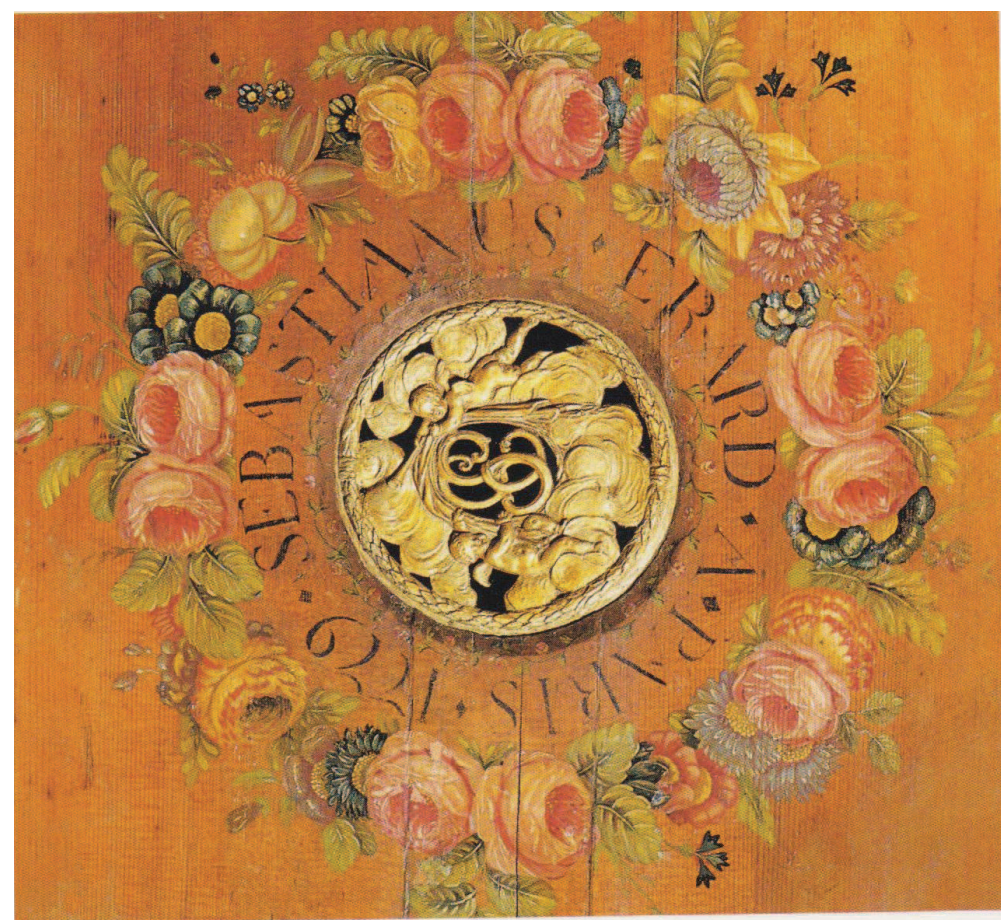

$A b b$. 7. Herstelleremblem in einem Rosenkranz auf einem mechanischen Clavecin mit der Inschrift: SEBASTIANUS ERARD A PARIS 1779; in der Mitte die Initiale von Genien 


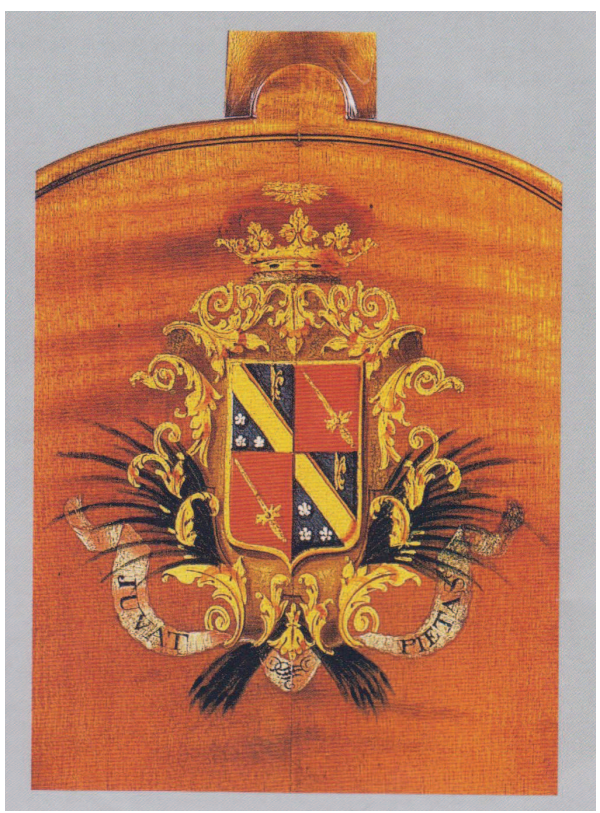

Abb. 8. Herstelleremblem des in seiner Zeit berühmtesten französischen Geigenbauers JeanBaptiste Vuillaume (1798-1875)

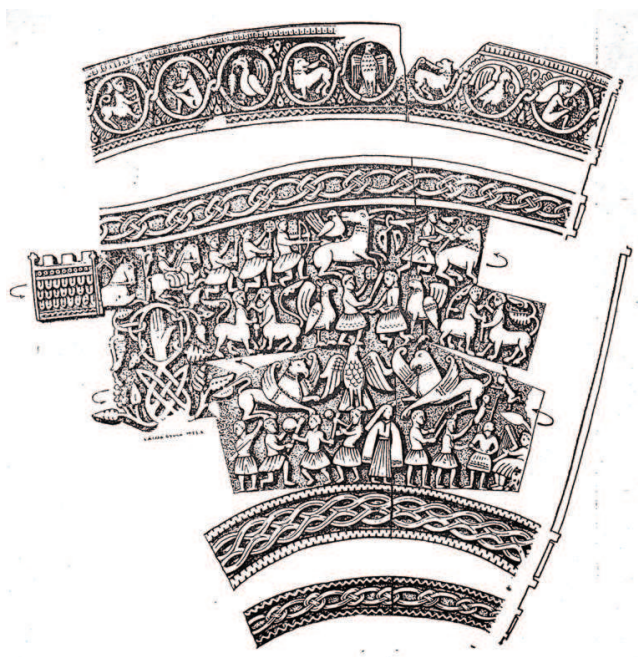

Abb. 9. Darstellungen der Spieler und Musizierenden auf dem sog. Lehel-Horn, Museum in Jászberény

tenbauers sind oft zugleich schöne visuelle Kompositionen, wie z. B. das von Jean-Baptiste Vuillaume (1798-1875), der alle seine Werke durchnummeriert hat (Abb. 8).

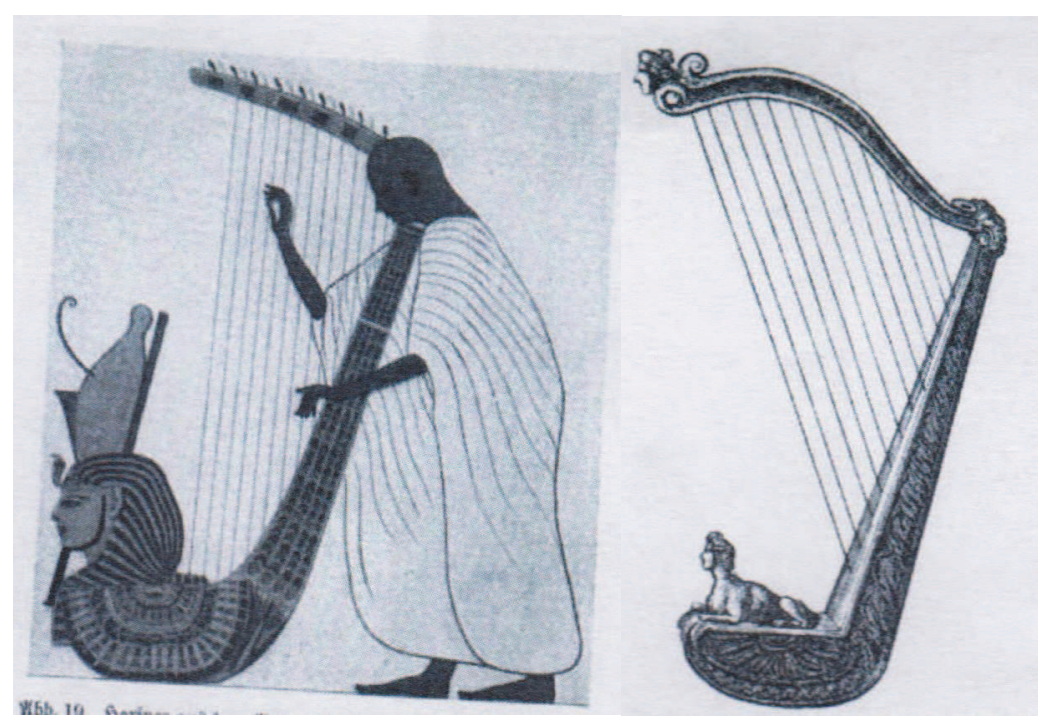

Abb. 10. Die „Ramses-Harfe“ nach Champollion (links) und nach Forkel (rechts) 
Beim Instrumentenbau bringen Material und Technik mitunter langzeitig gleiche Zeichen hervor. Anschaulich wird dieses Beispiel an den Knochenhörnern. Diese sind in der Regel verziert, und zwar gleichermaßen mit farbigen und ungefärbten Einkerbungen, Gravuren. Es kann sich um geometrische Abbildungen, Pflanzen-, Tier- und Menschendarstellungen, um einzelne Szenen oder gar um ganze Geschichten, Szenenreihen handeln. Ins Auge springt die Tatsache, dass mit ähnlicher Technik hergestellte andere Gegenstände (Trink- und Schießpulverhörner bzw. -dosen usw.) regelmäßig ganz ähnliche Verzierungen aufweisen. ${ }^{11}$ So kann eigentlich auch hierbei nicht von eigenständigen Musikzeichen gesprochen werden. Jedoch ist diese Art der Knochen-, Horn- und sogar Elfenbein-Verzierungswelt von der Kunst- und Kulturgeschichte auf zuverlässige Weise erschlossen und interpretiert worden.

Immerhin sollen wir festhalten, dass die Darstellung von Instrumenten und Musizieren an solchen Gegenständen und Musikinstrumenten häufig, ja betont vorkommt. Diese kann aber auch überaus komplex sein. Unter den Elfenbeinschnitzereifiguren des allgemein bekannten frühmittelalterlichen Lehel-Horns ${ }^{12}$ in Ungarn ${ }^{13}$ sind auch Harfenspieler und Hornist dargestellt, doch sind diese am wahrscheinlichsten Akteure einer Zirkusvorstellung - ebenso wie der Kraftmensch, der Balljongleur, ja selbst die dressierten Vögel unter den übrigen Darstellungen auf dem Horn (Abb. 9). Die Archäologen verweisen in der Regel darauf, dass es ursprünglich ein byzantinisches Musikinstrument für den Zirkus gewesen sein mag, wohl Anfang und Ende der Vorstellung oder einzelner Nummer anzuzeigen. Die Abbildung der Musiker an solchen Hörnern - uns ist noch ein Dutzend ähnlicher Exemplare aus dem zeitgenössischen Europa mit ähnlichen Darstellungen bekannt - ist mithin ein ikonisches Zeichen, das auf die Funktion des Signalinstruments verweist.

Nach alldem ist es immer noch überraschend, auf wie lange Vergangenheit die Gestaltung einzelner Musikinstrumente in symbolischen Formen zurückblicken kann. Selbst die heute gebräuchliche Harfe (mit Pedal) kann Züge aufweisen, als ob der Harfenrahmen eine bäuchlings liegende Figur symbolisierte. Über die Vergangenheit dieser Lösung stehen uns wahrhaftig frappierende Angaben zur Verfügung. Ein viel zitiertes Wandgemälde aus der altägyptischen Nekropolis zu Theben, datiert auf um 1166 v. Chr., zeigt den Pharao Ramses III. beim Spielen an einer Winkelharfe, die in einem Ägyptens Hoheitszeichen an sich tragenden Pharaonenkopf endet. ${ }^{14}$ Und dem können wir noch hinzufügen, dass einer der Begründer der wirklich universellen Musikgeschichte, Johann Nikolaus Forkel, als er im ersten Band seiner Allgemeinen Geschichte der Musik (Leipzig, 1788) dieselbe Harfe in einer Abbildung vorstellte, das Bild des Musikinstruments nach der einschlägigen Zeichenwelt und dem Geschmack seiner Zeit einfach umzeichnen ließ. Bei ihm ist oben an der Harfe ein Menschengesicht nach der Art der Gallionsfiguren zu sehen, unten sitzt eine bäuchlings liegende Sphynx ( $A b b$. 10). Das sind schon in jedem semiotischen Sinne Symbole: Jedoch nicht die des alten Ägyptens, sondern dem „Antiquitätsgefühl“ des Instruments (das ist also die Sphynx)

\footnotetext{
${ }^{11}$ FÉL-Hofer 1966.

${ }^{12}$ Genannt nach dem Stammesführer Lehel aus der ungarischen Landnahmezeit.

${ }^{13}$ LÁsZLó 1973.

${ }^{14}$ Siehe eine rekonstruierte farbige Illustration: Buchner 1995, Abb. 29 auf Seite 37.
} 
bzw. der meisterhaften Ausfertigung des Instruments (das ist der Kopf oben) entsprechend..$^{15}$

Der Symbolismus dieses Saiteninstruments lässt sich in noch größere vergleichende Perspektiven stellen. Die aus Mesopotamien stammende, nach der üblichen museologischen Rekonstruktion manchmal publizierte sumerische Lyra (aus Ur/Uruk, zwischen 2700 und 2500 v. Chr.) setzte an die Vorderseite des unteren Rahmens einen verzierten Stierkopf, versehen gar mit einem üppigen schwarzen Bart. ${ }^{16}$ An dem Rahmen von Saiteninstrumenten oder am Ende des Klangkörpers ist die Abbildung von Tierköpfen außerordentlich häufig. Am fantastisch verzierten Halsteil der so genannten „,mongolischen“ Geigen springt das am stärksten ins Auge. ${ }^{17}$ Am meisterhaft geschnitzten Kopfteil südslawischer Kniegeigen sind mitunter in mehreren Schichten, auf dem Rücken voneinander stehende Figuren angebracht: Reiter, Ochsen, sitzender Mann und häufig ein am meisten ziegenähnliches Tier mit langen Hörnern. ${ }^{18}$ Diese Abbildungen sind auch im semiotischen Sinne als Symbole zu betrachten.

Mitunter erscheint bei einer ganzen Gruppe von Musikinstrumenten die beinahe gleiche Symbolisation. Am meisten an jenen Streichinstrumenten im 17. Jahrhundert (wie cister, viola da gamba, viola da braccio und sogar Drehleier), bei denen der Kopfteil, an dem die Saiten befestigt werden, ein in einer Schneckenlinie endendes Element ist, kommen sehr häufig schön geformte, geschnitzte Frauenköpfe (seltener Männerköpfe), mitunter Frauenbüsten vor $(A b b$. 11). Die Tendenz der Darstellung ist klar. Der Hinweis auf Menschenkopf, Hals, gelegentlich auf hemdartige Kleidung, Tücher usw. ist eindeutig: Sie berufen sich auf die „Weiblichkeit“ der Musik. Auch diese Formenwelt können wir Symbol nennen, obgleich es gar nicht so einfach gesagt werden kann, was genau diese schön geschnitzten Köpfe signalisieren und warum sie an diesen Musikinstrumenten regelmäßig vorkommen, während z. B. an den gewöhnlichen Geigen selten vertreten sind.

Die beiden obigen Beispiele können wir gleich miteinander verknüpfen. Es ist allgemein bekannt, dass der Kopfteil des europäischen Dudelsacks sehr häufig zu einer regelrechten kleinen Skulptur geformt wird. ${ }^{19}$ Am bekanntesten ist der ziegenköpfige Dudelsack - hierbei können wir auf die bereits erwähnte stierköpfige sumerische Lyra oder die pferdeköpfige mongolische Geige zurückweisen. Wir kennen aber auch Dudelsackköpfe mit der Abbildung junger Bäuerinnen. Wobei die Frauenköpfe an den verschiedenen ,,viola“-artigen Instrumenten verhältnismäßig zierlicher und kleiner sind als die an

${ }^{15}$ Über die Probleme der Rekonstruktion der altägyptischen Harfe siehe: SACHS 1920. Auch die heutigen musikgeschichtlichen Illustrationen entsprechen dem Geschmack des Publikums. Die modernen Bilder verwechseln mal auch den rechten bzw. linken Teil der Illustration.

${ }^{16}$ Siehe eine Farbillustration: Buchner 1995, Abb. 25 auf Seite 33. Da die Holzteile usw. während der Jahrtausende verschwunden sind, hat man das Musikinstrument nur aus den metallenen Zierformen ,rekonstruiert" - mit vielen Fragezeichen.

${ }^{17}$ Siehe die Farbillustration einer mongolischen Morin-chur Geige: BuchNER 1995, Abb. 200 auf Seite 210. Der Name der mongolischen Pferdekopf-Geige, matouqin, stammt aus der chinesischen Sprache und bedeutet einfach „Musikinstrument mit Pferdekopf“.

${ }^{18}$ Siehe eine südslawische Gusle mit Reiter- und Ochsenfiguren: BuchnER 1995, Abb. 371 auf Seite 302.

${ }^{19}$ Die vielseitige ungarische Dudelsack-Fachliteratur (siehe zusammenfassend: Agócs 2001) erwähnt nur beiläufig den Anthropomorphismus der Instrumente. Aurél Szakál beschrieb die „menschenköpfigen“ Dudelsäcke von der Umgebung von Szeged: SzAKÁL 1992-1994. 


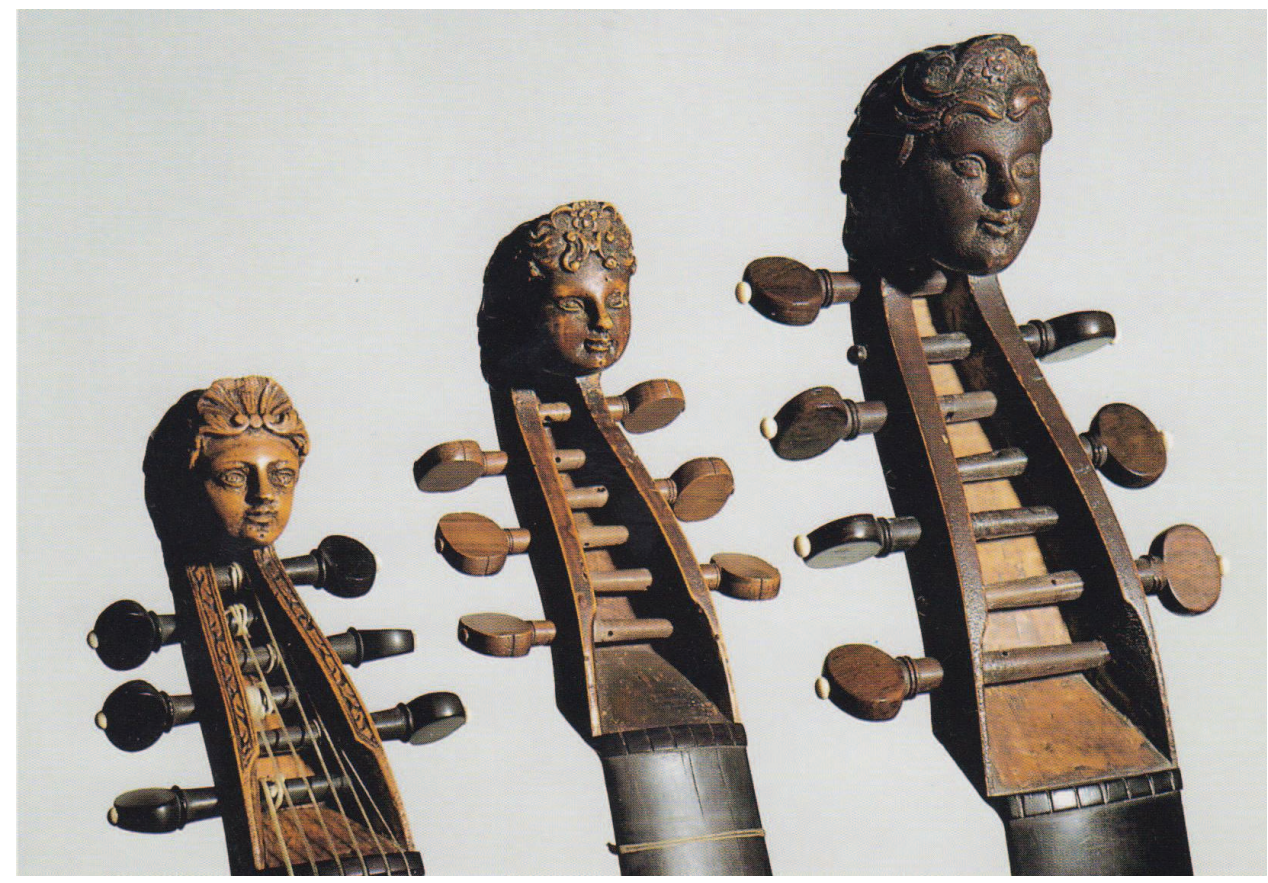

Abb. 11. Frauenköpfe an viole und basse de viole von Nicolas Bertrand. Paris, 1714, 1721, 1720

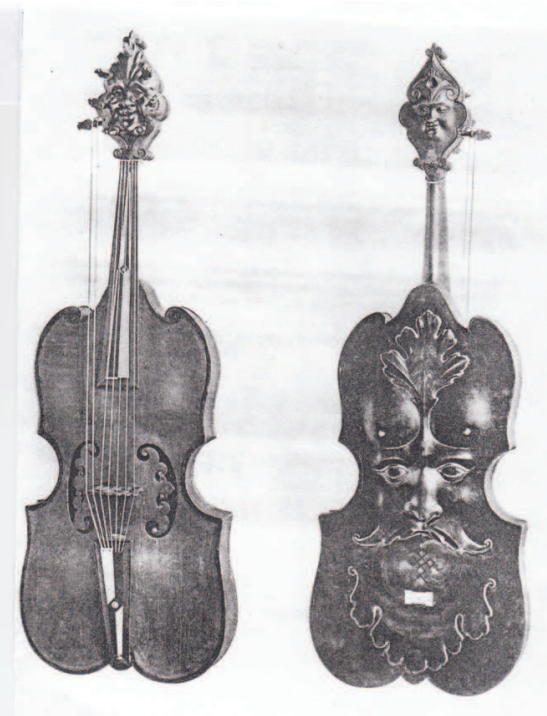

den Dudelsäcken. Hier sind weder die zoomorphen noch die anthropomorphen Instrumentenskulpturen zufällig. Dieser Symbolismus ist bewusst, ja selbst der duale Symbolismus von Tier- und Menschenkopf auf den Dudelsackdarstellungen ist dasselbe. Mit Vorliebe stellen europäische Dudelsack-Historiker die meisterhaften Miniaturen in den Manuskripten der um 1270 entstandenen Cantigas de Santa MariaKodexe vor. ${ }^{20}$ Hier sind sowohl Einpfeifen- als auch Zweipfeifen-Dudelsäcke zu sehen; diese werden von jeweils zwei, einander gegenüberstehenden oder sitzenden Spielleuten bedient. Auf allen Miniaturen ist an einem Dudelsack

Abb. 12. Vorderseite und Rückseite der Lira da braccio von Giovanni d'Andrea. Venedig, 1511

${ }^{20}$ Siehe die internationalen Publikationen des European Humanities Research Centre, University of Oxford „Cantigas de Santa Maria“ mit allen Bildern. 
ein gekröntes Menschenhaupt, am anderen ein Tierkopf zu sehen. Diese Symbolik ist scheinbar klar, ist jedoch wiederum nicht so einfach, wie es auch heutzutage noch viele annehmen würden. Der Dudelsack ist ein teuflisches, ein höllisches Musikinstrument und dieses Dämonische sollte der manchmal den Teufelsdarstellungen nahe kommende Ziegenkopf ausdrücken. Der gekrönte Männerkopf und der Tierkopf (bzw. deren Gegenüberstellung) symbolisieren gemeinsam und einander gegenübergestellt etwas, zu dessen semiotischer Erschließung weitere Forschungsarbeit betrieben werden müsste.

Dennoch beabsichtige ich nicht, meine zwangsläufig vorläufige Problemstellung pessimistisch $\mathrm{zu}$ beenden - wenngleich es ersichtlich ist, dass wir ,in vielem“ mit der eigentlichen Forschung eben noch angefangen hatten. Wenn wir die Verzierung der Musikinstrumente unter semiotischem Aspekt untersuchen, soll festgehalten werden, dass die Wichtigkeit dieses Problemkomple-

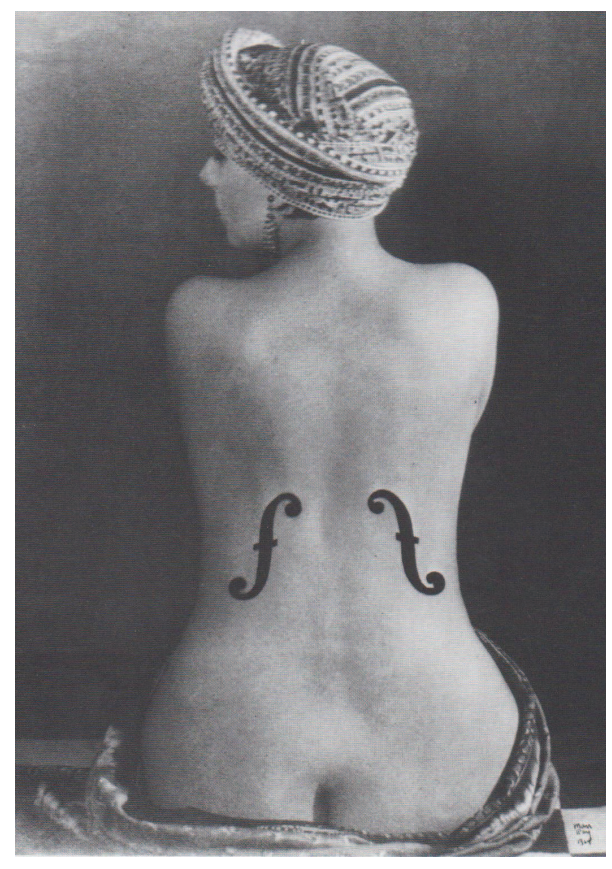

Abb. 13. Le violon d'Ingres, Foto von Man Ray. Paris, 1924 xes bereits vom Klassiker der ungarischen und europäischen Ethnomusikologie, László Lajtha, klar formuliert wurde. In seiner 1929 veröffentlichten Schrift, ${ }^{21}$ die in einer der Kunst, d. h. nicht dem Gegenstand Musik gewidmeten Zeitschrift erschien, erörtert er das Problem auf einer bis zum heutigen Tag gültigen, sachlichen Weise. Anders als er damals können wir es nicht einmal heute formulieren. ${ }^{22}$

Für die weitere Forschung und die Interdisziplinarität führe ich nur ein Beispiel an: Der menschliche Körper als Musikinstrument reicht von der Lira da braccio von Giovanni d'Andrea (Venedig 1551) (Ab $^{23}$. 12) bis zu Man Ray's Le violon d'Ingres ${ }^{24}$ (Abb. 13).

${ }^{21}$ LAJTHA 1929; siehe jetzt: LaJTHa 1992: 202-213.

${ }^{22} \mathrm{Zu}$ den wenigen bahnbrechenden Studien in Ungarn gehören: HARASZTI 1926 und FürsT 1943.

${ }^{23}$ In der letzten Zeit hat man die Identität der geigenartigen Instrumenten vom 16. Jahrhundert stark bezweifelt - für die antrophomorphe Darstellung es beweisst noch stärker die Anwenbarkeit der Semiotik in der Musikgeschichte.

${ }^{24}$ Zur Ikonographie der Musik im Allgemeinen: Crane 1971; Brown-Lascelle 1972; und besonders die RIdIM (Répertoire International d'Iconographie Musicale). 


\section{LITERATUR}

Agócs, Gergely (szerk.)

2001: A duda, a furulya és a kanásztülök. A magyar hangszeres zene folklórja. Budapest.

BAINES, Anthony (ed.)

1992: The Oxford Companion to Musical Instruments. London.

Brown, Howard Mayer - LASCElle, Joan

1972: Musical Iconography. A Manuel for cataloguing of musical subjects in the Western art before 1800.

Harvard.

BuCHNER, Alexander

1995: Handbuch der Musik-Instrumente. Hanau. (3. Auflage)

CRANE, Frederick

1971: A Bibliography of the Iconography of Music. University of Iowa.

FéL, Edit - HoFER, Tamás

1966: Husaren, Hirten, Heilige. Menschendarstellungen in der ungarischen Volkskunst. Budapest.

FüRST, Leontin

1943: Hangszerek a magyar képzőmüvészetben. (A Budapesti Királyi Magyar Pázmány Péter Tudományegyetem Müvészettörténeti és Keresztényrégészeti Intézetének dolgozatai 83.) Budapest.

HaraszTi, Emil

1926: Hangutánzás és jelentésváltozás az egyetemes és a magyar hangszertörténetben. Budapest.

JoNES, Sterling Scott

1995: The lira da Braccio. Bloomington-Indianapolis.

Kaden, Christian - Brachmann , Jan - Giese, Detlef

1998: Zeichen. In: Die Musik in Geschichte und Gegenwart. Sachteil. Bd. 9. Kassel. 2149-2220.

LaJTHa, László

1929: Diszitett hangszerek. Magyar Mủvészet V. évf. 3. szám, 132-141. = Lajtha László összegyüjtött irásai. I. Budapest, 1992. 202-213.

LÁszLó, Gyula

1973: Lehel kürtje. Jászberény. (Negyedik kiadás)

Marcel-Dubois, Claudie

1980: L'instrument de musique populaire. Usage et symboles. Paris.

MidgLEy, Ruth

1978: Les instruments de musique du monde entier. Paris.

NöTH, Winfried

2000: Handbuch der Semiotik. Stuttgart-Weimar. (2., vollständig neu bearbeitete und erweiterte Auflage)

Posner, Roland - Robering, Klaus - SEBeOK, Thomas A. (Hrsg.)

1997-2004: Semiotik. Ein Handbuch zu den zeichentheoretischen Grundlagen von Natur und Kultur / Semiotics. A Handbook on the Sign-theoretic Foundation of Nature and Culture. I-IV. Berlin-New York.

SACHS, Curt

1920: Altägyptische Musikinstrumente. Leipzig.

1930: Handbuch der Musikinstrumentenkunde. Leipzig.

SAdie, Stanley (ed.)

1984: The New Grove Dictionary of Musical Instruments. I-III. London.

SCHAFFNER, André

1936: Origine des instruments de musique. Introduction ethnologique à l'histoire de la musique. Paris.

(Auch als spätere Nachdrucke.)

Sebeok, Thomas A. - Danesi, Marcel (eds)

2010: Encyclopedic Dictionary of Semiotics. I-III. Berlin-New York. (3rd edition)

SZAKÁL, Aurél

1992-1994: A szegedi menyecskefejes duda. In: Zenetudományi Dolgozatok, 199-216.

1993: Emberfejes dudafejek a Magyar Alföld déli részén. In: Móra Ferenc Múzeum Évkönyve 1991-92/1: 153-173, LXII-LXX. 
TARASTI, Eero

1994: A Theory of Musical Semiotics. Bloomington.

VAN DER MEER, John Henry

1983: Musikinstrumente. München. (In ungarischer Übersetzung: Hangszerek az ókortól napjainkig. Budapest, 1988.)

Voigt, Vilmos

2008: Bevezetés a szemiotikába. Budapest.

Wade-MatThews, Max

2009: The World Encyclopedia of Musical Instruments. London.

WINTERNITZ, Emanuel

1967: Musical Instruments and their Symbolism in Western Art. New York. 